\title{
The Cognitive Engineering of Memory in Educational Website Design
}

\author{
Jessica Jones \\ Department of Psychology \\ Liverpool Hope University, United Kingdom \\ Jill Fitzpatrick \\ Department of Psychology \\ Liverpool Hope University, United Kingdom \\ Philippe Chassy \\ Department of Psychology \\ Liverpool Hope University, United Kingdom
}

\begin{abstract}
The ability to recall items from memory is often vital, whether for remembering a phone number, or when sitting an examination. Memory also affects our aesthetical judgements of objects, and the ways in which we perceive such objects as complex or simple. The role of memory likewise extends to our interactions with computer technology; influencing our overall experience of websites, the processing of information provided by websites and thus the quality of our learning processes. For many people, the internet is their primary source of information, and hence websites designed to facilitate learning should be designed in line with the cognitive architecture of the user. This paper exposes the main components of human memory, outlining its limits. We then consider the role of three memory-related factors: prototypicality, visual complexity, and aesthetics, on the user's interaction with a website. A key point of the present article is that websites should be designed with consideration to the constraints of human memory. Finally, we emphasise how such limits should be respected, and how effectively capitalising on the memory structure of the user should aid efficient learning.
\end{abstract}

Keywords: memory, human-computer interactions, website design, prototypicality, visual complexity, aesthetics.

\section{THE COGNITIVE ENGINEERING OF MEMORY IN EDUCATIONAL WEBSITE DESIGN}

In 2014, around 2.92 billion people used the internet worldwide; a colossal rise from the reported 394 million in 2010 [1]. For many people, accessing the internet is part of everyday life, for example for work or social purposes. The internet has likewise become increasingly important within education [2]. Basic computer skills are now acquired as early as preschool, in order to develop spatial and problem-solving skills in young children [3]. In formal education, the move from textbooks alone to a combination of internet and computer-assisted learning offers a series of advantages, including increased efficiency, enhanced accessibility, and reduced costs [4]. Computer assisted learning is also increasingly common at university level education, with many nursing and medicine courses offering computer-assisted instruction as part of the regular curriculum [5]. The field of Human-Computer Interaction (HCI) is concerned with the ways in which people engage with computer technology [6]. Since the internet is crucial to the daily activities of many people, a major focus of HCI research has been on websites specifically [7]. In this paper, we highlight the limits of the architecture of 
memory which impinge on the user's experience. We start by exposing the main components of human memory, and then move on to discuss the influence of three specific memory-related factors on website design and usability: prototypicality, complexity, and aesthetics.

Memory plays a crucial role in the everyday lives of people. Without memory, everything would be a novel experience, the world and its events would appear unpredictable, and longterm learning or knowledge acquisition would not take place. Long term memory is one's database of acquired information; the place where all knowledge and skills accumulated over a lifetime are stored. Whenever a piece of knowledge is required it is retrieved from long-term memory and placed within working memory. Working memory is the psychological space wherein readily accessible information is processed. It holds our conscious representation of the external world, and drives attention to important aspects of reality. By simulating the evolution of events, we are able to make decisions based upon the information held within working memory, thus enabling the anticipation of action consequences. Working memory is central to the conductance of tasks as it enables the processing, manipulation and updating of current information [8-9]; vital for the comprehension and calculation of events and situations. Several models of working memory have been developed [10-11]. Among them, the model of Baddeley [12], which conceives of working memory as a set of four interconnected components, is the most prominent. Within the model, a limited-capacity attentional controller known as the central executive is supported by two slave systems, the phonological loop which holds verbal information, and the visuospatial sketchpad, dealing with visual information only. The fourth element, the episodic buffer, acts as a temporary interface between the central executive, its slave systems, and long-term memory. In doing so, it is capable of combining and storing the multidimensional information from these components as integrated chunks, thus binding several types of information into whole objects and episodes. It is the episodic buffer which holds the current metal, conscious representation of the world.

While working memory as proposed by Baddeley enables the efficient processing of large amounts of information, it is constrained by certain boundaries. For instance, an integral finding in human cognition is that the capacity of working memory is limited to around seven simultaneous chunks of information [13]. Although the exact number of available chunks has been disputed [14-15] it is nonetheless certain that working memory is not boundless. A chunk space in working memory can be occupied by several types of information, where each space is occupied by either a singular aspect, or by a unit of information comprised of a combination of elements. For example, in the case of verbal information, the letters $U, S$, and A would occupy three spaces in working memory if one has no experience of that particular order of those particular letters before. If however, one is familiar with the acronym commonly used for the United States of America, the information can be stored much more efficiently, fusing the separate letters into one unit, which is thereby stored as one chunk: USA. Our understanding of reality and our capacity to capture information depends upon the architecture of human cognition, in particular on the limited capacity of memory. Therefore, human-designed learning systems should take such limits into account in order to optimize the learning experience of students. This is especially true for purely computer-based learning, which by definition lacks the human teacher's wide repertoire of adaptive behaviour.

The influence of memory on our learning experience is pivotal. For example, ample experience of a particular object or situation results in the construction of a concept of that object or situation in long-term memory [16]. The concept is considered as the prototype, against which all instances are assessed in terms of similarity [17]. The prototypicality of an object is thus considered as the degree to which it is representative of the general mental model or template 
of that class of object in memory [18]. Due to the presence of the prototype in memory, current relevant information can be processed without considerable impingement on working memory capacity. As such, prototypical stimuli are processed fluently [19]. Research on the eye movements of expert chess players provides support for efficient processing of prototypical stimuli, as they are able to rapidly detect complex patterns due to the presence of existing perceptual chunks stored in memory. Likewise, the knowledge-guided perception of chess experts has been documented through the ability of the expert to attend rapidly to the most important features of a position [20]. Prototypicality has also been shown to have an effect on an object's perceived attractiveness; for example, preference for highly prototypical stimuli has been documented in studies on facial attractiveness, where average faces are deemed more attractive than those that are more unusual [21-22]. Similar findings have been obtained using various stimuli including for example cubist paintings [23] and colours [24]. It has been suggested that the positive reaction to a prototypical stimulus is a consequence of its fluency in processing [19]. That is, objects of high prototypicality correspond highly with the stored prototype, and hence are processed more efficiently and favourably than objects that are further away from the match. The influence of prototypicality on the facilitation of information processing has also been investigated in websites. A study by Tuch et al. [25] found that users preferred prototypical websites when judging aesthetics. Web page designs that contradicted a user's memory-generated expectation had a negative effect on their first impressions, implying that both prototypicality and visual complexity are vital components of website construction [25-26]. Hence, a website with a structure which matches the memory prototype held by the user should undoubtedly enhance the learning experience.

Website design is thus a matter of concern for both computer scientists and psychologists. It is crucial that websites are designed in line with the actual memory structure of users, reflecting the mental categories that the learner uses to structure reality. Ideally, good pedagogic practice would involve profiling user knowledge prior to design. Typically however, experts create material which matches their mental structures, which unfortunately, learners commonly do not share. As a consequence, learners quickly become lost with the material. For instance, statistical packages constitute a central aspect of learning in psychology but are not designed for learner use. The software provides too much information, and in this case, memory cannot cope with recently learned information, new information, and the structure of the software. Similarly, many websites designed to teach statistics are structured by expert statisticians or expert psychologists. Whilst such websites match the knowledge of psychologists, for learners, the discrepancy between the ways in which they have been taught and the structure of the site impedes usability. As an example, many students are taught to consider the design of the experiment in order to determine the type of test to apply. A cognitively engineered website would offer choices as a function of the design; however, many present a basic list of statistical tests: leaving the choice of the test to the user. Despite the fact that the explanation of each test is provided, the website structure differs from the user's memory structure, and thus expectations, hence resulting in an incompatibility that the user experiences difficulty in solving.

Since information is interpreted through memory structures, it is evident that the amount of information captured at any one time depends upon the knowledge of the individual. Complexity is thus the result of information overflow in human memory. Visual complexity has been well documented within HCI, as ample research has demonstrated its role in the usability of websites [27-30]. Although a robust definition has proved difficult throughout the literature [31], the term is applicable to and has been measured by subjective appraisals of complexity 
and objective aspects concerned with the physical properties of stimuli, such as number of elements [32] or image compression size [33]. Specifically, studies show that the degree to which a website is perceived as complex influences user interaction [30]. Design features such as font, colour, and layout affect the visual processing of information and can impact upon users' motivations for navigation [34-35]; their overall response as consumers [28]; and likelihood of revisiting a site [36]. Thus web designers should be aware of the significance of establishing an ideal scale of complexity for the optimal user-website connection.

Memory also has a crucial effect on the aesthetic experience of individuals [37]. Classic research on aesthetics describes the relationship between aesthetical pleasure and visual complexity in the form of an inverted-U curve, where the optimum level of pleasure corresponds to a moderate degree of complexity [32]. Preference for moderate levels of complexity has likewise been documented in websites [38]. Similarly a study with children has found that websites of medium complexity are preferable to those of low or high levels [27]. A further investigation with children also reports an overall preference for moderate complexity [39]. That is, the peak point of preference concerns stimuli complex enough to engage the perceiver, but not so complex as to cause overload. Despite such findings however, many studies conducted on websites have yielded a negative linear relationship between complexity and aesthetics [25, 40]. A negative relationship has also been discovered through psychophysiological measures, where visual complexity is associated with negative valence appraisal and an increase in facial muscle tension [28]. A more recent study also reports a negative relationship between the two measures [41]. Moreover, an investigation conducted using smartphone interfaces has found a strong relationship between simplicity and user satisfaction [42]. Taken together, these results suggest that objects deemed simple or moderately complex, as assessed by memory, provide the best user experience.

Aesthetical appeal, as emerging from memory processing, is also an important aspect of web design. Investigations into the aesthetical properties of websites [25, 28, 38, 40, 43], reflect this. For instance, the aesthetical properties of websites have been shown to impact on a variety of aspects such as user trust [44], intentions to revisit a site[45], and user satisfaction [46]. Aesthetical appraisal is evaluated rapidly, as users judge the attractiveness of a website in as little as 50 milliseconds [47]. As mentioned, research indicates that the attractiveness of a stimulus depend on both its perceived level of complexity [30] and depends both upon its perceived level of complexity [28] and upon the similarity to its classification in memory [25]. A likely preference for highly prototypical and thus low visually complex stimuli finds support through the negative linear relationship between visual complexity and aesthetical pleasure found in a substantial portion of the research conducted on websites [40-41]. Indeed, Tuch et al. [25] have reported a relationship between visual complexity and prototypicality as determinants of aesthetical appraisal, as highest ratings were typically obtained from sites both low in complexity and high in prototypicality.

While arguably all websites would be wise to capitalise on the knowledge provided by $\mathrm{HCI}$ and cognition research to enhance the efficient processing of information and elicit positive appraisals, this is of particular relevance to websites designed for educational purposes. The acquired knowledge on perceived complexity is useful for the design of better user interfaces and well-structured websites. Visual complexity, which is actually a consequence of memory [48], will emerge for the user once information overloads working memory capacity. It is thus essential that educational websites contain only the most vital information necessary for the learner. Parsing the information into segments, and ensuring that each new segment is learned properly (via repetitive exercises) before progressing on to more information is essential to a 
good learning process and hence to website design. For example, boxes can be used containing optional information for the advanced learner, but the complexity of information should not vary in a given piece of text, at the risk of the learner getting lost in the details. Regarding a website's aesthetical value, perceived attractiveness is not only important for appraisal but also plays a role in maintaining user engagement and improving the user's experience. In light of the fact that users can often spend hours on educational websites, design should aim to improve the perceptual experience of the user in order to effectively facilitate learning.

\section{References}

1. Office for National Statistics. Internet access - households and individuals. 2014 [cited 20151 April]; Available from: www.ons.gov.uk.

2. Gross, E., Adolescent internet use: what we expect, what teens report. Journal of Applied Developmental Psychology, 2004. 25: p. 633-649.

3. Sandberg, A., Preschool teacher's conceptions of computers and play. Information Technology in Childhood Education, 2002. 2002(1): p. 245-262.

4. Bybee, R.W. and L.W. Trowbridge. The future of computer and internet use in schools. 2014 [cited 20151 April]; Available from: www.education.com.

5. Greenhalgh, T., Computer assisted learning in undergraduate medical education. BMJ, 2001. 322: p. 40-44.

6. Velásquez, J.D., Combining eye-tracking technologies with web usage mining for identifying Website Keyobjects. Engineering Applications of Artificial Intelligence, 2013. 26(5-6): p. 1469-1478.

7. Hasan, L. and E. Abuelrub, Assessing the quality of web sites. Applied Computing and Informatics, 2011. 9(1): p. 11-29.

8. Baddeley, A., The episodic buffer: a new component of working memory. Trends in Cognitive Science, 2000. 4: p. 417-423.

9. Baddeley, A., Working memory. Science, 1992. 255: p. 556-559.

10. Owen, A.M. and A.C. Evans, Evidence for a two-stage model of spatial working memory processing within the lateral frontal cortex: a positron emission tomography study. Cerebral Cortex, 1996. 6: p. 31-38.

11. Byrne, M.D. and S. Bovair, A working memory model of a common procedural error. Cognitive Science, 1997. 21: p. 31-61.

12. Baddeley, A., The episodic buffer: a new component of working memory? Trends in Cognitive Sciences, 2000. 4(11): p. 417-423.

13. Miller, G.A., The magical number seven. Plus or minus two: some limits on our capacity for processing information. Psychological Review, 1956. 63(2): p. 81-97.

14. Cowan, N., The magical number 4 in short-term memory: a reconsideration of mental storage capacity. Behavioral and Brain Sciences, 2001. 24: p. 87-114.

15. Gobet, F. and G. Clarkson, Chunks in expert memory: evidence for the magic number four... or is it two? Memory, 2004. 12: p. 732-747.

16. Rosch, E.H., Natural categories. Cognitive Psychology, 1973. 4: p. 328-350.

17. Rosch, E. and C.B. Mervis, Family Resemblances: Studies in the Internal Structure of Categories. Cognitive Psychology, 1975. 7(4): p. 573-605.

18. Leder, H., et al., A model of aesthetic appreciation and aesthetic judgments. British Journal of Psychology, 2004. 95: p. 489-508.

19. Winkielman, P., et al., Prototypes Are Attractive Because They Are Easy on the Mind. Psychological Science, 2006. 17: p. 799-806.

20. De Groot, A.D. and F. Gobet, Perception and memory in chess: Heuristics of the professional eye1996, Assen: Van Gorcum. 
21. Etcoff, N., Survival of the Prettiest: The Science of Beauty1999, New York: Anchor Books, Doubleday.

22. Langlois, J.H. and L.A. Roggman, Attractive faces are only average. Psychological Science, 1990. 1: p. 115121.

23. Hekkert, P. and P.C.W. Wieringen, Complexity and prototypicality as determinants of the appraisal of cubist paintings. British Journal of Psychology, 1990. 4: p. 483-513.

24. Martindale, C., The pleasure of thought: A theory of cognitive hedonics. Journal of Mind and Behavior, 1984. 5: p. 49-80.

25. Tuch, A.N., et al., The role of visual complexity and prototypicality regarding first impression of websites: Working towards understanding aesthetic judgments. International Journal of Human-Computer Studies, 2012. 70(11): p. 794-811.

26. Roth, S.P., et al., Mental models for web objects: Where do users expect to find the most frequent objects in online shops, news portals, and company web pages? Interacting with Computers, 2010. 22(2): p. 140152.

27. Wang, H.F. and C.J. Bowerman. The impact of perceived visual complexity on children's websites in relation to classical and expressive aesthetics. in IADIS international conference IADIS interfaces and human computer interaction 2012. 2012. Lisbon: Inderscience Publishers.

28. Tuch, A.N., et al., Visual complexity of websites: Effects on users' experience, physiology, performance, and memory. International Journal of Human-Computer Studies, 2009. 67(9): p. 703-715.

29. Deng, L. and M.S. Poole, Aesthetic design of e-commerce web pages - Webpage Complexity, Order and preference. Electronic Commerce Research and Applications, 2012.11(4): p. 420-440.

30. Mai, R., et al., The shifting range of optimal web site complexity. Journal of Interactive Marketing, 2014. 28(2): p. 101-116.

31. Xing, J. and C. Manning, Complexity and automation displays of air traffic control: Literature review and analysis.Technical Report, 2005, US Department of Transportation, Office of Aerospace Medicine.

32. Berlyne, D.E., Aesthetics and Psychobiology1971, New York: Century-Crofts.

33. Riglis, E., Modeling visual complexity in image architectures. Technical Report., 1998, Heriot-Watt University.

34. Cuddihy, E. and J.H. Spyridakis, The effect of visual design and placement of intra-article navigation schemes on reading comprehension and website user perceptions. Computers in Human Behavior, 2012. 28(4): p. 1399-1409.

35. Saadé, G.R. and C. A. Otrakji, First impressions last a lifetime: effect of interface type on disorientation and cognitive load. Computers in Human Behavior, 2007. 23(1): p. 525-535.

36. Wang, Q., et al., An eye-tracking study of website complexity from cognitive load perspective. Decision Support Systems, 2014. 62(0): p. 1-10.

37. Sherman, A., et al., Visual-object working memory affects aesthetic judgments. Journal of Vision, 2013. 13: p. 1308.

38. Geissler, G.L., G.M. Zinkhan, and R.T. Watson, The influence of home page complexity on consumer attention, attitudes, and purchase intent. Journal of Advertising, 2006. 35(2): p. 69-80.

39. Wang, H.-F., Picture perfect: Girls' and boys' preferences towards visual complexity in children's websites. Computers in Human Behavior, 2014. 31: p. 551-557.

40. Pandir, M. and J. Knight, Homepage aesthetics: the search for preference factors and the challenges of subjectivity. Interacting with Computers in Human Behavior, 2006. 18: p. 1351-1370.

41. Chassy, P., et al., The negative relationship between visual complexity and aesthetical pleasure, in Annual Conference of the British Psychological Society2014: Birmingham.

42. Choi, J.H. and H.J. Lee, Facets of simplicity for the smartphone interface: A structural model. International Journal of Human-Computer Studies, 2012. 70: p. 129-142.

43. van Schaik, P. and J. Ling, The role of context in perceptions of the aesthetics of web pages over time. International Journal of Human-Computer Studies, 2009. 67: p. 79-89. 
44. Karvonen, K., The beauty of simplicity. Proceedings on the 2000 conference on universal usability. The association for computing machinery, 2000: p. 85-90.

45. Mahlke, S., Factors influencing the experience of website usage, in Extended Abstracts on Human Factors in Computing Systems2002, ACM Press: New York. p. 846-847.

46. Tractinsky, N., A.S. Katz, and D. Ikar, What is beautiful is usable. Interacting with Computers, 2000. 13: p. 127-145.

47. Lindgaard, G., et al., Attention web designers: you have 50 milliseconds to make a good first impression! Behaviour and Information Technology, 2006. 25(2): p. 115-126.

48. Chassy, P. and F. Gobet, Visual search in ecological and non-ecological displays: Evidence for a nonmonotonic effect of complexity on performance. PLoS ONE, 2013. 8(1): p. e53420. 